[DRAFT NOTE - 26 JANUARY 2021]

\title{
How Do Different Frames Affect Public Support for Climate Change Policy: Evidence from a Multi-Country Conjoint Study
}

\author{
Niheer Dasandi', Hilary Grahami, David Hudson', Slava Jankin Mikhayloviii, Jennifer \\ vanHeerde-Hudson ${ }^{\text {iv }} \&$ Nick Watts ${ }^{v}$
}

\begin{abstract}
This study tests the effects of different framings of climate change messages on public support for mitigation policy using conjoint survey experiments conducted in five countries: China, Germany, India, the UK, and the USA. We consider four different types of climate change frames: valence (positive vs. negative), theme (economic, environmental, health, and migration), scale (individual, community, country, and global), and timeframe (2050, 2030, now). The analysis also tests the effects of these different frames on individuals who are not concerned by the effects of climate change. Our results show a positive framing, in terms of the opportunities they provide, increases support for mitigation policies. We also find that an environmental and health framing of climate change increases public support for mitigation, while a migration framing reduces public support, and an economic framing has no effect. The results also show that framing climate change at a global level elicit greater public support for mitigation than at an individual level, and discussing the current impacts of climate change leads to greater support than future impacts. Our analysis also finds that a positive framing and a health framing of climate change also increase support for mitigation policies among those not concerned by the effects of climate change.
\end{abstract}

\section{Introduction}

The Paris Agreement aims to hold the increase in global temperature to well below the critical $2^{\circ} \mathrm{C}$ threshold to reduce the risks and impacts of climate change. Negotiated the $21^{\text {st }}$ Conference of the Parties of the UN Framework Convention on Climate Change (UNFCCC), it instituted an inclusive approach in which all state parties to the Agreement made non-binding

\footnotetext{
' International Development Department, University of Birmingham. Please email any comments or questions to Niheer Dasandi at n.dasandi@bham.ac.uk.

ii Department of Health Sciences, University of York

iii Data Science Lab, Hertie School, Berlin

iv Department of Political Science, University College London

${ }^{v}$ Institute for Global Health, University College London
} 
commitments to emissions reduction. Countries decide on their own contributions to achieving a low-emissions pathways and climate resilient development, set out in their Nationally Determined Contributions (NDCs), to be reviewed and revised every five years. ${ }^{1}$

For such a non-binding approach based on countries setting their own contributions to be effective, it is important that the public is engaged with, and supports, policies to address climate change. Indeed, levels of public support can have an important effect on governments' mitigation targets. ${ }^{2}$ Such public buy-in is regarded as essential for the long-term success of the Paris Agreement. With pledges made in the 2015 set of NDCs falling well short of containing global temperature increases to below $2^{\circ} \mathrm{C}$, there is an urgent need to strengthen the commitments countries make in the 2020 round of enhanced NDCs, which in turn requires greater public awareness and support for mitigation.

This has led to renewed attention to the challenge of communicating climate change in ways that build public support for mitigation policies. ${ }^{3}$ There has been particular interest in how framing climate change messages influences engagement. ${ }^{4}$ Studies have considered how different types of frames impact public support for climate policies. Some have considered whether a positive framing of climate change messaging, in terms of focusing on the opportunities and benefits of initiatives to tackle climate change, rather than negative framings that emphasise the threat climate change poses can increase public support. ${ }^{5}$ Others have considered how framing climate change according to different issues or themes can impact public engagement. For example, the growing awareness of the health impacts of climate change have led some to argue that emphasising the effects of climate change on people's health may increase public support for mitigation policies. ${ }^{6,7}$ The impact of other themes, such as an economic framing of climate change, on public attitudes have also been considered. ${ }^{8} \mathrm{~A}$ further issue is whether the impacts of climate change are framed in local or global terms. Some find that emphasising local rather than global impacts increases support for climate change policies ${ }^{9}$, while others suggest that local climate change impacts are perceived as less severe than global impacts. ${ }^{10}$ Another aspect of framing is the timeframes used in climate change messaging, with studies looking at whether people are more likely to support climate change policies in order to protect future generations compared to addressing current impacts. ${ }^{1112}$ While various studies have considered these different types of frames separately, few have examined the effects of these different climate change frames 
on public support for mitigation policies together to reveal the relative importance of the different frames.

This study addresses this gap. We consider how different frames in climate change communication impact public support for mitigation policies around the world using an experimental research design. We conducted conjoint survey experiments in five countries China, Germany, India, the UK, and the USA - in September 2020. Our study tests how different types of frames impact public support for climate policy, by assessing the effects of valence, theme, scale, and time frames on public support for mitigation policies. As such, the study looks at how these different frames impact support for climate policy in the five countries.

In doing so, we address two further issues in the existing literature. The first is that much of the existing research on public attitudes to climate change policies and framing climate change messages is focused on high-income countries, particularly the USA, with far less attention given to countries in the Global South. ${ }^{13}$ Our study looks at five countries - China, Germany, India, the UK, and the USA. By considering how these various frames affect public support for mitigation policies in these countries, we can better understand the relationship between climate change communication and public attitudes in different contexts across the world.

The second issue that this study addresses is how such framing of climate change messages influences support for climate policy among those who are not concerned by the effects of climate change. There has been growing attention to the question of how to motivate proenvironmental behaviour among climate change sceptics and deniers ${ }^{14}$, with some studies pointing to growing polarisation between climate change 'convinced' and 'sceptics' whereby they tend to engage in different debates. ${ }^{15}$ To address this issue, we also examine the types of frames that may increase support for mitigation policies among those unconcerned by climate change.

The results of our analysis have important implications for climate change communication:

1. We find that framing climate change policies in terms of the opportunities they provide increases public support for mitigation policies, while emphasising the threat climate change poses tends to reduce support for such policies. 
2. Our analysis shows that employing an environmental or public health frame also increases support for climate policies. In contrast, we find that using an economic frame has no significant effect on public support for mitigation, and using a migration frame has a negative effect on public support for climate policies.

3. The results also suggest that discussing the global impact of climate change tends to elicit greater public support for mitigation, while focusing on the individual or community impacts tends to reduce support.

4. The results also show that discussing the impacts that climate change has now tends to generate more public support for climate policies than discussing future impacts.

5. Our analysis also considers the types of frames that may be most effective in eliciting support among those unconcerned by the effects of climate change. We find that a positive framing of climate change policies, in terms of the opportunities they provide, along with a health framing of climate change, increase support for mitigation policies among those who are not concerned with the effects of climate change.

The analysis also identifies important country differences in these findings demonstrating the importance of understanding specific country contexts in designing effective climate change communication.

\section{Methods}

Our analysis draws on conjoint survey experiments conducted in five countries: China, Germany, India, the UK and USA. The surveys in Germany, the UK and the USA are based on nationally representative samples. In China and India, we use samples that are representative of urban populations in the two countries. The survey experiments were conducted in the five countries in September 2020. The total sample size was 7,512 with a sample of at least 1,500 in each country. ${ }^{\mathrm{vi}}$

In the conjoint surveys, participants were asked to read two randomly generated statements about climate change, and to indicate which of the two statements would make them more

\footnotetext{
vi The exact sample sizes for each of the five countries are as follows: China, $n=1,502$; Germany, $n=1,501 ;$ India, $n=1,506$; UK, $n=1,500$; and USA, n-1,503.
} 
likely to support policies to tackle climate change, as Figure 1 shows. Each participant received five pairs of randomly generated statements, which varied according to the different frames.

\begin{tabular}{|} 
Statement A \\
Climate change is the greatest \\
threat facing the world right now \\
due to the health impacts. This \\
includes rising temperatures and \\
more frequent extreme weather \\
events increasing the spread of \\
infectious disease and worsening \\
well-being. This is making things \\
worse for the world.
\end{tabular}

\begin{tabular}{l} 
Statement B \\
Tackling climate change is the great \\
opportunity for the world right now \\
due to the migration benefits. This \\
includes investing in regions that \\
are most impacted by the effects of \\
climate change to help ensure \\
people can continue to live and \\
work in these regions. This is \\
making things better for the world. \\
\hline
\end{tabular}

Figure 1. Examples of randomly generated statements in the conjoint surveys.

The statements vary according to four different frames: valence, theme, scale, and time. For each of these frames, there were a number of different variations included. The valence frame included two variations - framing the impacts of climate change as an opportunity or a threat. The theme frame included four different themes: economic, environmental, health, and migration. The message would include a reference to the theme frame as well as an example of how climate change is related to the particular theme (see Figure 1). The scale frame included four different options: personal, community, country, and world. Finally, the timeframe included three different variations: 2050, 2030, and now. This means that in total there were 96 different variations of the statement, with participants being asked to compare pairs of randomly generated statements (as in Figure 1), five times.

In addition to participants being asked to select which statements would make them more likely to support climate change policies, they were asked standard socio-demographic questions (e.g., gender, income, education, etc.). Furthermore, earlier in the survey, before the conjoint experiment, they were asked to express how concerned they are about the impacts of climate change. 


\section{Results and Analysis}

In this section, we present preliminary results of the conjoint experiments. We first consider the findings across the five countries, before we examine the differences in the results between the five countries. We then focus, in particular, on the effects of the different frames between those people that express concern about climate change and those that are not concerned about the effects of climate change.

\section{Overall Results}

In Figure 2, we present the results of the conjoint analysis from all five countries together. The results suggest that people are more supportive of policies to address climate change when they are presented as opportunities rather than threats. Hence, we find that the positive framing of climate change policy is more effective at garnering support than the negative framing. In terms of the theme frames, we find that two of the frames have a positive effect on support for climate change policies: the environmental frame and the health frame. In contrast, we find that the migration frame significantly reduces support for mitigation policies, and the economic framing has no significant effect on support for policies to address climate change across the five countries. Therefore, when participants are presented with the environmental and health impacts of climate change, they are more likely to support mitigation policies. However, when presented with the migration impacts of climate change, we find that participants are less supportive of such policies, while being informed about the economic impacts of climate change has no effect on people's support for climate change policies. 


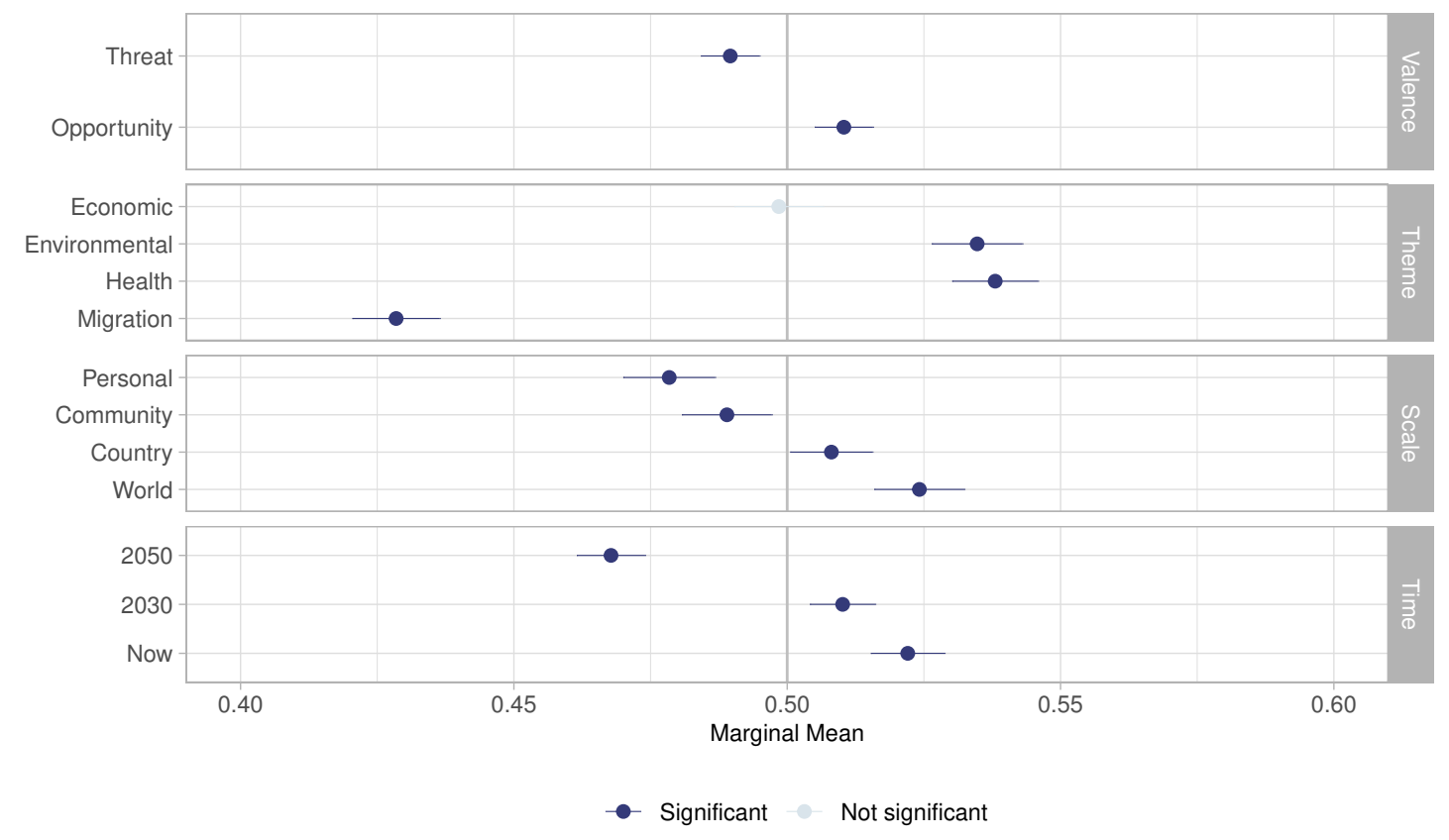

Figure 2. Results of conjoint survey experiments pooled for all five countries. The results that are statistically significant at the 0.05 level are shown in the darker colour, and results that are not statistically significant are shown in grey.

Figure 2 also presents the effects of using different scales and time frames on people's support for mitigations policies. We find that support for policies to tackle climate change increases as we move from an individual to global scale. When climate change is framed as having an impact on the participant, personally, or on the participant's community, they are less supportive of policies to tackle climate change. In contrast, they are more supportive of such policies when the impacts of climate change on their country are discussed, and participants were most supportive when presented with a message about the impacts of climate change on the world. Hence, we find that discussing national and global impacts of climate change is more likely to elicit support for mitigation policies than community or individual-based messages. The results suggest that people are more supportive of policies to tackle climate change when presented with a message that discusses the impacts of climate change in the present rather than in the future. The results show that discussing the impacts that climate change will have in 2050 leads people to be less supportive of mitigation policies. In contrast when the messages discuss the impacts of climate change ten years from now (2030) or now, participants were more supportive of policies to address climate change, with the latter having the largest positive effect. 


\section{Individual Country Results}

We next consider the differences in the results of the conjoint experiments across the five countries. Figure 3 presents the results for China, Germany, India, the UK, and the USA. The results confirm that framing climate change as an opportunity tends to lead to greater support for policies to tackle climate change than presenting it as a threat. This effect is largest in China, and can also be observed in the UK and the USA. In India, we find that the valence of framing does not significantly impact public support for mitigation policies. Furthermore, in Germany we find the opposite effect; framing climate change as a threat elicits greater support for mitigation policies than framing it as an opportunity.

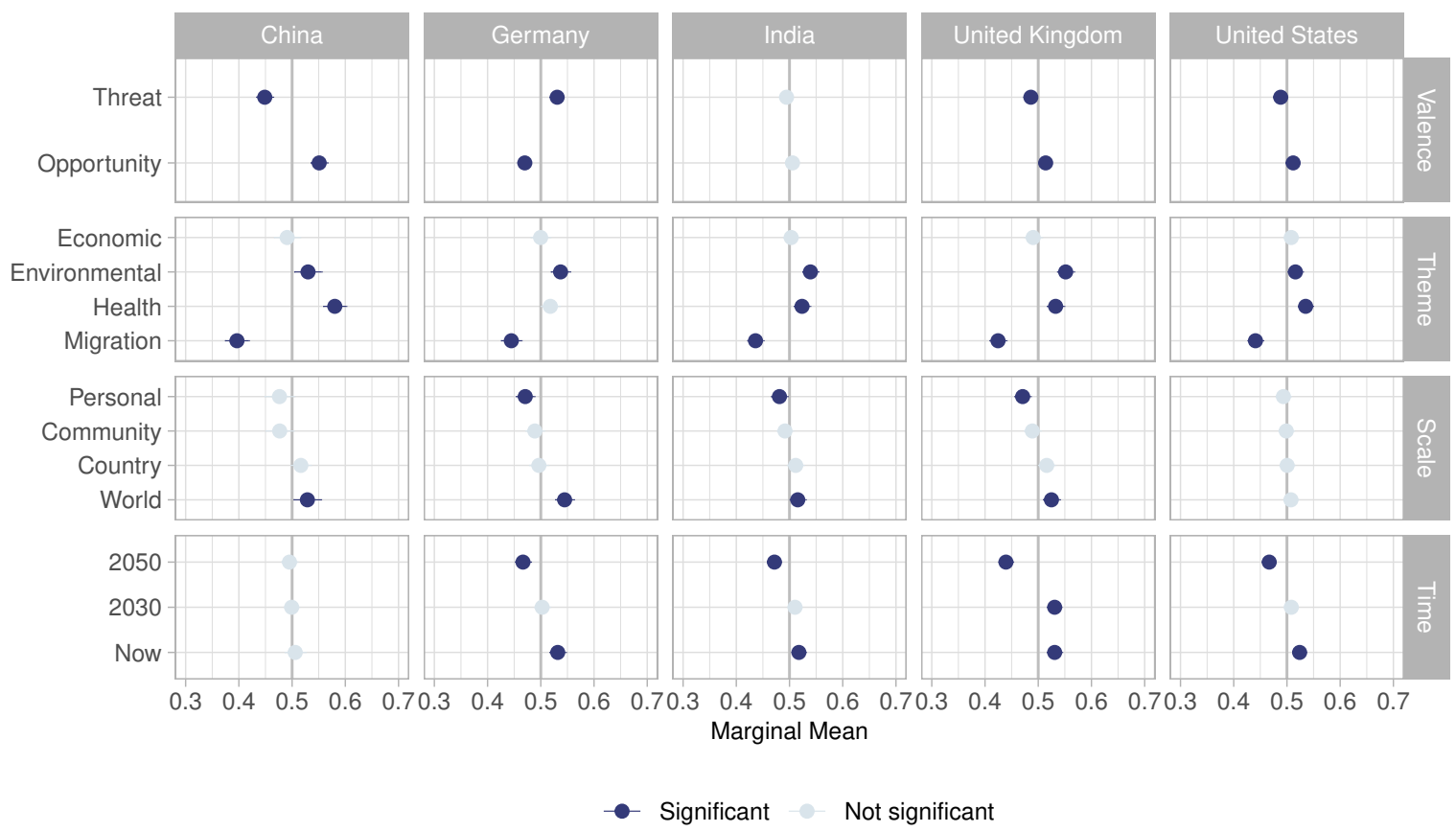

Figure 3. Results of conjoint survey experiment for China, Germany, India, the UK and USA. The results that are statistically significant at the 0.05 level are shown in the darker colour, and results that are not statistically significant are shown in grey.

When we turn to the theme frame, we find that the economic framing has no effect on public support for policies to tackle climate change in all five countries. We find that the environmental frame has a positive effect on support for mitigation policies in four of the countries - China, Germany, India, and the UK - but has no significant effect on support for 
such policies in the USA. We find that the health framing has a positive effect on public support for policies to tackle climate change in all of the countries except Germany (the effect among participants in Germany falls below 0.05 confidence level). We find that the migration frame has a large negative effect on public support for mitigation policies in each of the five countries.

\section{Results for Those Not Concerned with Climate Change}

In many countries, there are segments of the public that express scepticism regarding the negative impacts of climate change. Therefore, an important issue when looking at public support for mitigation policies is whether particular frames are able to elicit support for such policies among those less concerned by the effects of climate change. In the survey experiments we conducted in each of the countries, we asked participants about the extent to which they were concerned with climate change. Figure 4 shows the proportion of participants for each level of concern in the five countries. The results show that climate scepticism is highest in the USA, with almost $25 \%$ of participants stating they are not at all or not very concerned about climate change. In Germany and the UK, around 16\% of participants stated they are not at all or not very concerned, while in China and India less than $7 \%$ of participants stated they are not at all or not very concerned about climate change. The highest levels of concern regarding climate change can be observed in India and China, though it is again worth noting that our sample of participants in these two countries is representative of the urban populations of these two countries. 


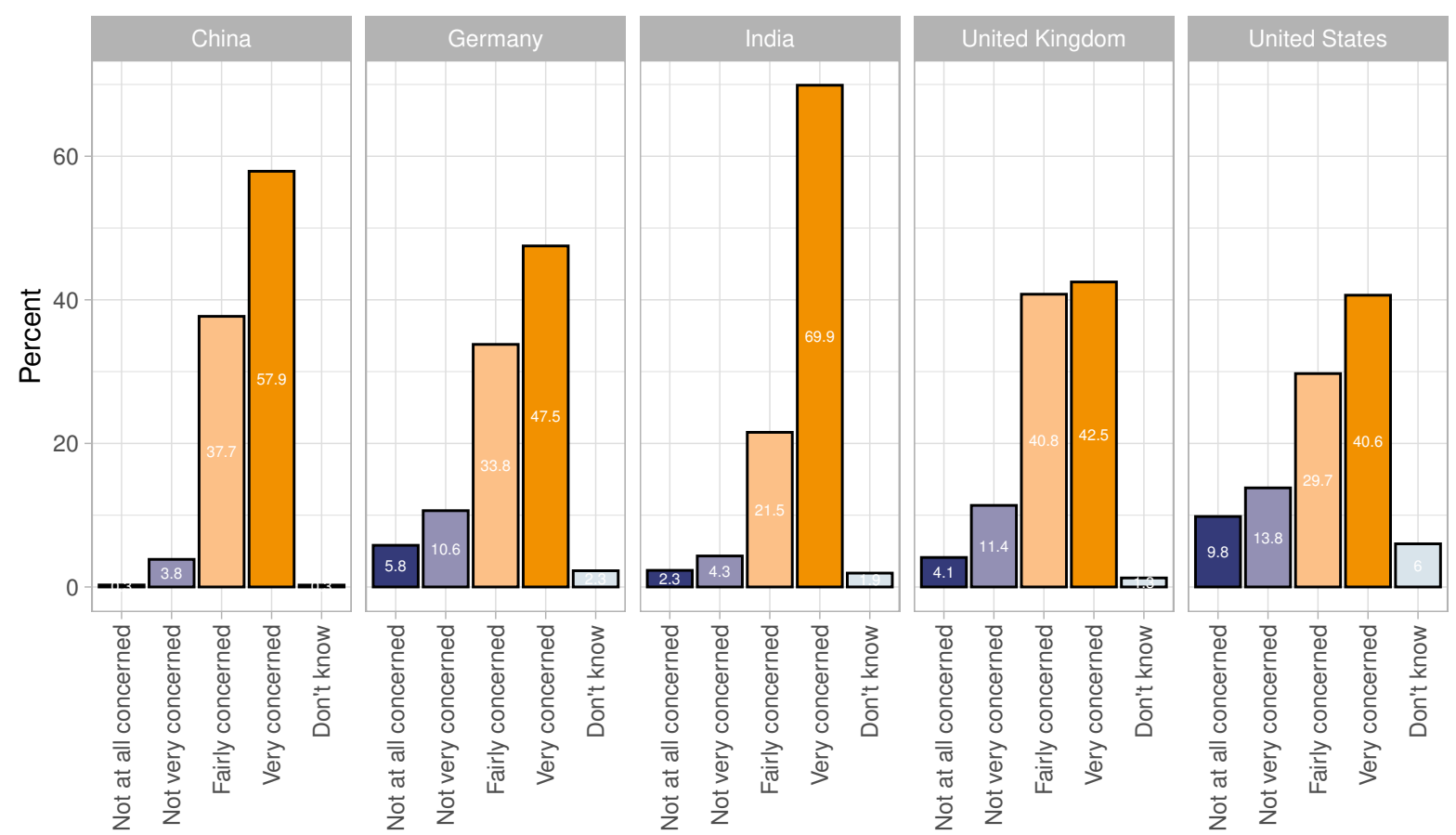

Figure 4. Proportion of participants at different levels of concern for climate change in each country.

We consider the results of the conjoint experiments by comparing those participants who say that they are fairly or very concerned about the effects of climate change with those that stated they are not at all or not very concerned about the impact of climate change. In Figure 5 we present the overall results from all five surveys comparing the results for the 'not at all' and 'not very' concerned ('Not concerned' in Figure 5 and 6) with those that are 'fairly' or 'very' concerned ('Concerned' in Figure 5 and 6). Figure 6 shows these results for each of the five countries. 


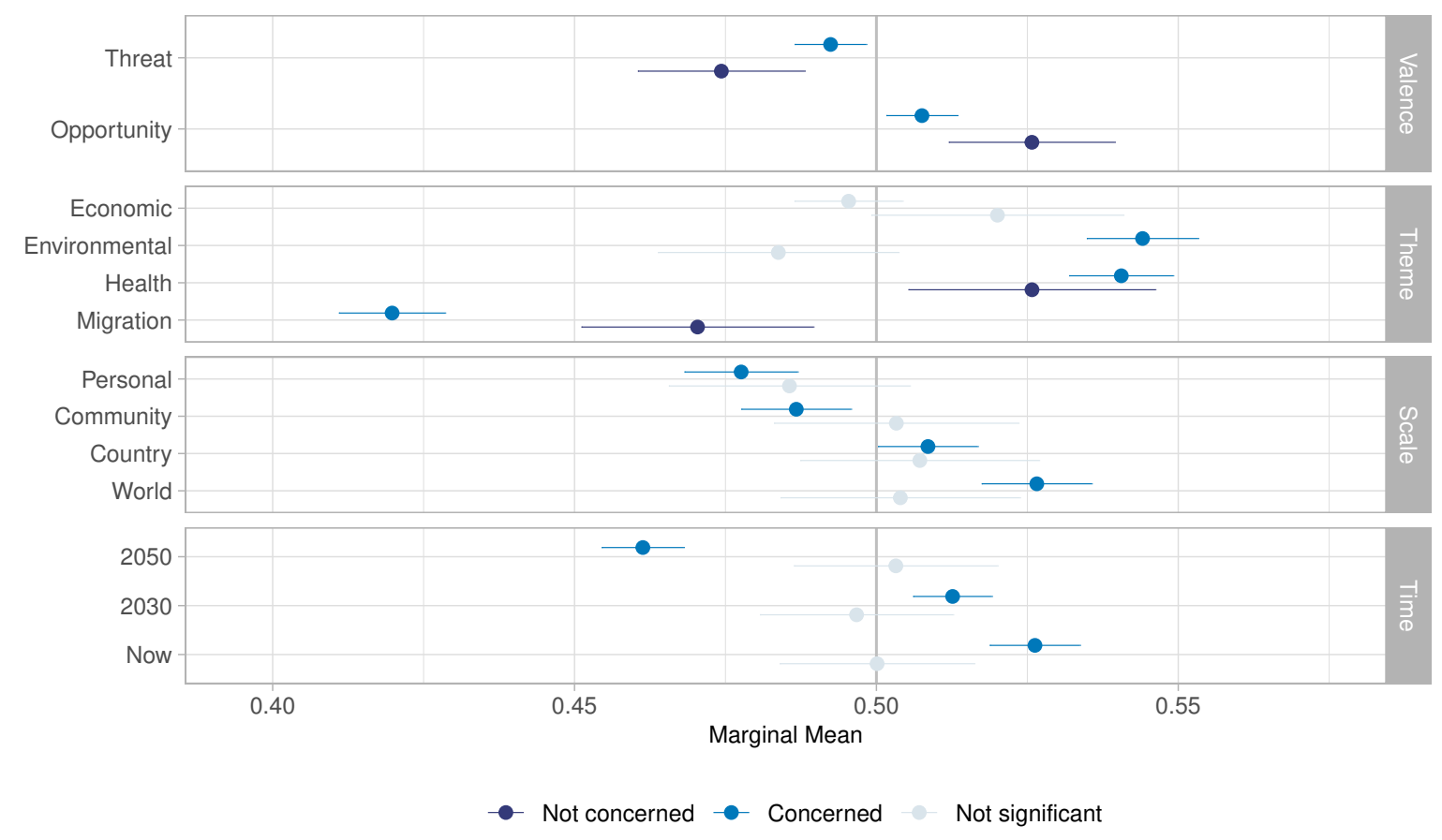

Sample size $n=7,512$ | Data are weighted to be nationally representative | Fieldwwork by Deltapoll, September 2020 Question: Respondents were shown two statements in which the valence, theme, scale, and time
frame were varied at random, providing 96 possible permutations. Respondents were asked: Indicate which of the two statementis would make you more likely to support policies to tackle climate change.

Figure 5. Results of conjoint survey experiments pooled for all five countries comparing participants that are not concerned about the effect of climate change with those that express some concern. The results that are statistically significant at the 0.05 level are shown in the darker colour, and results that are not statistically significant are shown in grey.

As we would expect, Figure 5 shows that in general we find that most of the frames do not impact support for mitigation policies amongst those not concerned with climate change. There are, however, two important exceptions to this. We find that those not concerned with the negative effects of climate change are more supportive of mitigation policies when climate change is presented as an opportunity, and less supportive when it is presented as a threat. Furthermore, we find that when a health framing is used, participants who are not concerned with climate change are more supportive of mitigation policies. In Figure 6, we present the individual country breakdown of these results, though it is worth stating that given the much smaller number of participants that express no or little concern about climate change in each country, there is much lower statistical power in this analysis, and hence more statistically insignificant results. Figure 6 suggests that our findings on the effect of positive valence on support for climate policies among those not concerned by climate change is largely driven by results in the UK and USA, and the finding on the effects of the health framing is largely driven by results in China and the USA. 

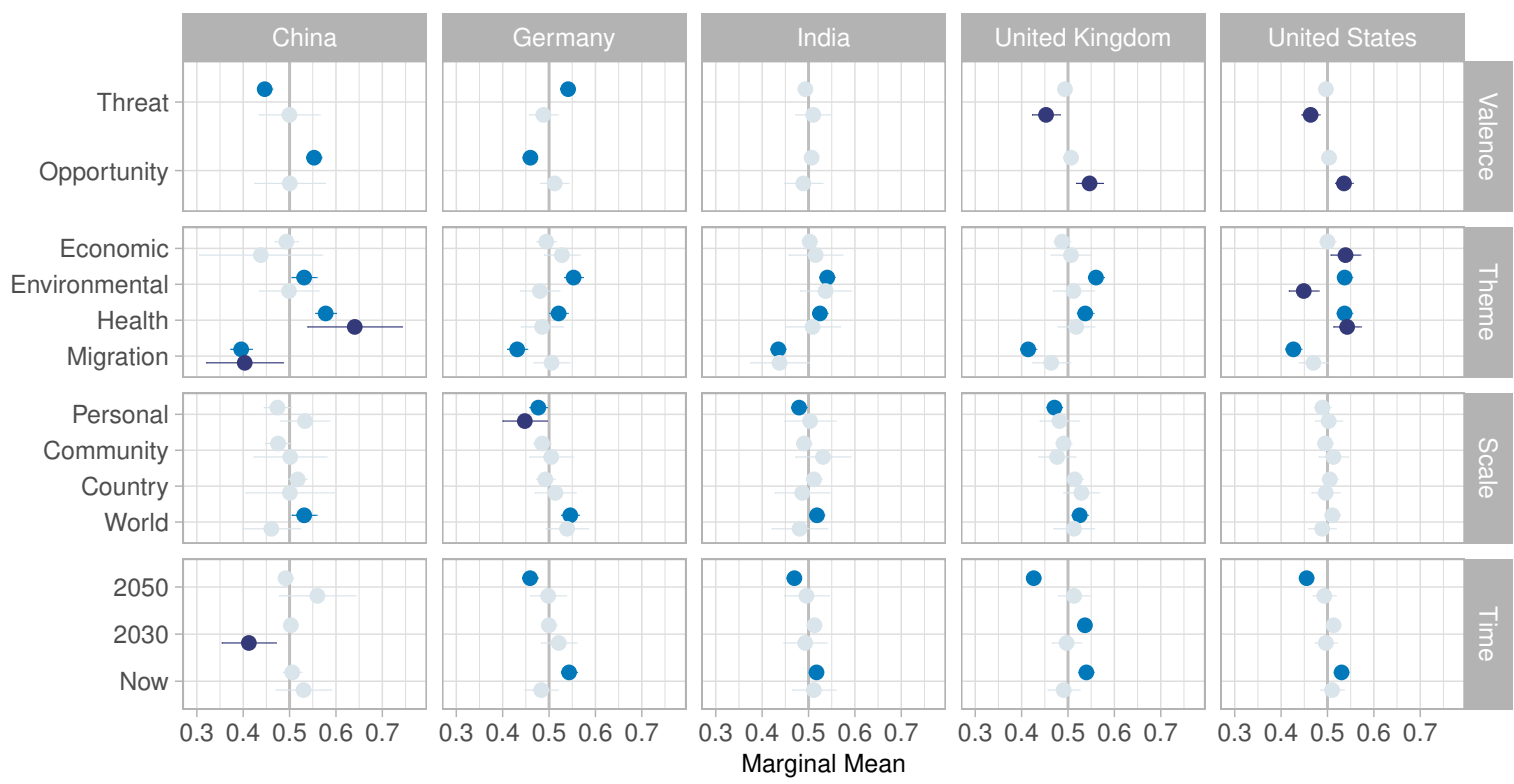

-- Not concerned

- Concerned

Not significant

Sample size: China $n=1,502$, Germany $n=1,501$, United Kingdom $n=1,500$, India=1,506, United States $n=1,503$ | Data are weighted to be nationally representative | Fieldiwork by Deltapoll, September 2020 Questic Respondents were shown two statements in which the valence, theme, schide
would make you more likely to support policies to tackle climate change.

Figure 6. Results of conjoint survey experiment for China, Germany, India, the UK and USA comparing participants that are not concerned about the effect of climate change with those that express some concern. The results that are statistically significant at the 0.05 level are shown in the darker colour, and results that are not statistically significant are shown in grey.

\section{Conclusion}

This study has considered how framing climate change in different ways impacts public support for climate policies through the use of conjoint survey experiments in China, Germany, India, the UK and USA. Specifically, we consider four different types of frames in climate change communication - valence, theme, scale, and time. Furthermore, we consider how results vary between participants who express no concern about climate change and those who express some concern. As noted earlier, our survey experiments were conducted at a single point in time, and significantly this was more than six months into the COVID-19 pandemic. Given the strong contextual influences on public support for climate policies, repeating the surveys is an important area of future research to ensure that these findings are consistent over time.

Our analysis provides general findings that can be observed across different countries, as well as important country differences. We find that overall a positive framing of climate change 
policies, in terms of the opportunities they provide, leads to greater public support for mitigation policies compared to framing climate change as a threat. However, our results suggests that the opposite is true in Germany, where threats are more effective at eliciting support for climate policies. The results also suggest that framing climate change in terms of its environmental and health impacts leads to greater public support for mitigation policies. In contrast, we find that an economic frame has no significant impact on public support for climate policies, and in all five countries we find that emphasising the effects of climate change on migration has a negative effect on public support for climate policy.

The analysis also sheds important light on how different frames can lead to support for climate policies among those not concerned with the effects of climate change. We find that that emphasising the opportunities that climate change policies provide can lead to support for such policies among those not concerned with climate change. Furthermore, we find that emphasising the health dimensions of climate change can also elicit support for mitigation policies among those not concerned about climate change. The findings of the study provide important new evidence on how the framing of climate change messages in different ways can impact public support for mitigation policies from around the world.

\footnotetext{
${ }^{1}$ Tobin P, Schmidt NM, Tosun J, Burns C. 2018. Mapping states' Paris climate pledges: Analysing targets and groups at COP 21. Global Environmental Change 48: 11-21.

2 Drummond A, Hall, LC, Sauer, JD, and Palmer MA. 2018. Is public awareness and perceived threat of climate change associated with governmental mitigation targets. Climatic Change 149: 159-171.

${ }^{3}$ Moser SC. 2010. Reflections on climate change communication research and practice in the second decade of the $21^{\text {st }}$ century: what more is there to say? Wiley Interdisciplinary Reviews: Climate Change 7(3): 345-369.

${ }^{4}$ Nisbet MC. 2009. Communicating climate change. Why frames matter for public engagement. Environment: Science and Policy for Sustainable Development 51(2): 12-23.

${ }^{5}$ Bernauer T and McGrath L. 2016. Simple reframing unlikely to boost public support for climate policy. Nature Climate Change 6(7): 680-683.

${ }^{6}$ Watts N, et al. 2018. The Lancet Countdown on Health and Climate Change: From 25 Years of Inaction to a Global Transformation for Public Health. The Lancet 391: 581-630.

${ }^{7}$ Maibach EW, Nisbet M, Baldwin P, Akerlof K, and Diao G. 2010. Reframing climate change as a public health issue: an exploratory study of public reactions. BMC Public Health 10(1): 299.

${ }^{8}$ Spence A, Pidgeon N. 2010. Framing and communicating climate change: The effects of distance and outcome frame manipulations. Global Environmental Change 20: 656-667.

${ }^{9}$ Wiest SL, Raymond L, Clawson RA. 2015. Framing, partisan predispositions, and public opinion on climate change. Global Environmental Change 31: 187-198.

${ }^{10}$ Spence A, Pidgeon N. 2010. Framing and communicating climate change: The effects of distance and outcome frame manipulations. Global Environmental Change 20: 656-667.

${ }^{11}$ Graham H, de Bell S, Hanley, N, Harvis, S, and White PCL. 2019. Willingness to pay for policies to reduce future deaths from climate change: evidence from a British survey. Public Health 174: 110-117.
} 
${ }^{12}$ Graham H, Bland M, Cookson R, Kanaan M, and White PCL. 2017. Do people favour policies that protect future generations? Evidence from a British survey of adults. Journal of Social Policicy 46(3): 423-445.

${ }^{13}$ Capstick S, Whitmarsh L, Poortinga W, Pidgeon N, and Upham P. 2015. International trends in public perceptions of climate change over the past quarter century. WIREs Climate Change 6: 35-61.

${ }^{14}$ Bain PG, Hornsey MJ, Bongiorno R, and Jeffries C. 2012. Promoting pro-environmental action in climate change deniers. Nature Climate Change 2(8): 600-603.

${ }^{15}$ Hoffman AJ. 2011. Talking past each other? Cultural framing of skeptical and convinced logics in the climate change debate. Organization \& Environment 24(1): 3-33. 